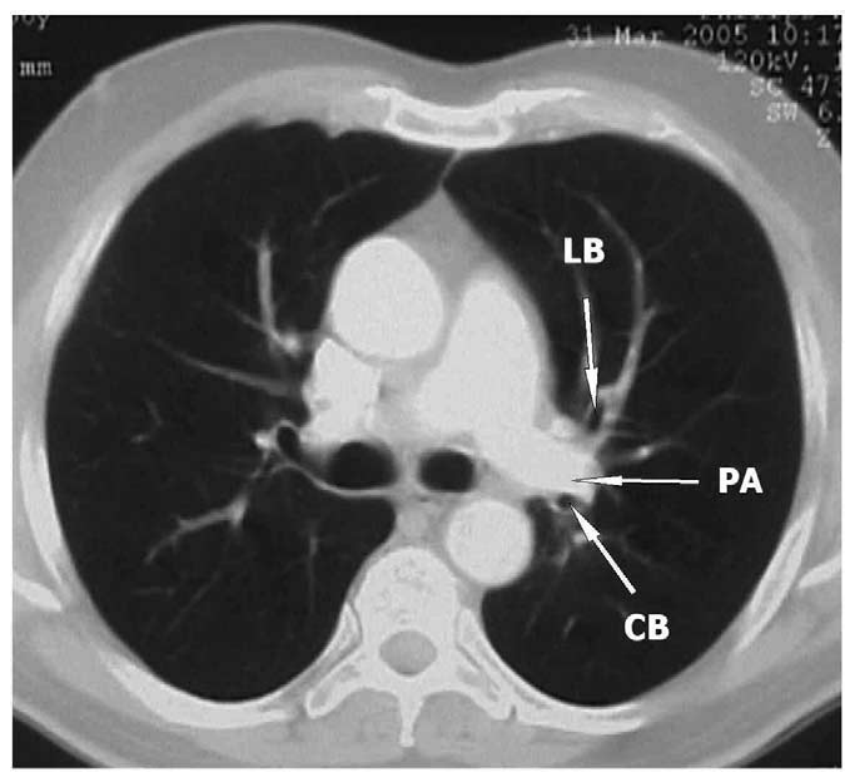

Figure 2. Preoperative computed tomographic scan of the chest showing the anomaly of the course of the left pulmonary artery. $P A$, Pulmonary artery; $C B$, common bronchus to the anterior and apical posterior segments; $L B$, lingular bronchus. this was confirmed by the preoperative bronchoscopy, which showed normal anatomy in both cases.

In our 2 patients the anomaly of the course of the left pulmonary artery precluded adequate dissection of the origin of the left upper bronchus and mandated separate closure of the common bronchus to the anterior and apical posterior segments and the lingular bronchus. In this particular anatomic situation, the interposition of neighboring tissue, such as pericardial fat, is a precautionary measure to prevent postoperative bronchovascular fistulas.

This anatomic variation was not identified preoperatively in either of the 2 patients. However, on review of the computed tomographic scan, the arterial anomaly could be clearly identified (Figure 2).

In conclusion, the particular anatomic variation of the left pulmonary artery described in this article, unassociated with bronchial anomalies, seems to demonstrate a relatively high frequency. Detailed knowledge of this vascular anomaly and its potential presence is therefore important for the training and practicing thoracic surgeon.

\section{Reference}

1. Galetta D, Debrosse D, Gossot D, Serra M, Grunenwald D. Anomalous left bronchial segmentation associated with an abnormal left pulmonary artery course. Ann Thorac Surg. 2004;78:e16.

\title{
A modified elephant trunk technique: The 3-fold elephant trunk technique
}

Kenji Okada, MD, Taijiro Sueda, MD, Kazumasa Orihashi, MD, Katsuhiko Imai, MD, and Katsutoshi Sato, MD, Hiroshima, Japan

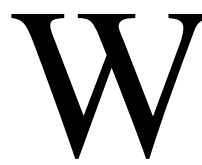

e report a modified elephant trunk (ET) technique, the 3-fold ET technique. This technique is an alternative for preventing thromboembolic events and paraplegia.

\section{Technical Description}

The reversed and bidirectional $\mathrm{ET}^{1}$ is made by invaginating the prosthesis. The idea for this modified technique arose from envelop-

\footnotetext{
From the Department of Cardiovascular Surgery, Hiroshima University Hospital, Hiroshima, Japan.

Received for publication July 6, 2005; accepted for publication Aug 3, 2005.

Address for reprints: Kenji Okada, MD, Department of Cardiovascular Surgery, Hiroshima University Hospital, 1-2-3 Kasumi, Minami-ku, Hiroshima, 734-8551, Japan (E-mail: kokada@ hiroshima-u.ac.jp).

J Thorac Cardiovasc Surg 2005;130:1720-2

$0022-5223 / \$ 30.00$

Copyright $\odot 2005$ by The American Association for Thoracic Surgery doi:10.1016/j.jtcvs.2005.08.011
}

ing the prosthesis instead of invaginating it. A single and straight prosthesis is folded twice and made into a 3 -fold ET (Figure 1). The first fold is placed about $7 \mathrm{~cm}$ from the end. The second fold is made backward, $5 \mathrm{~cm}$ back to the proximal site. In other words, the distance between the proximal edge and the second fold is about $2 \mathrm{~cm}$. Two stitches are placed on each of the proximal and distal sides to fix the ET in place. The stitches are put in to prevent the invasion of blood flow into the fold. The ET is about $7 \mathrm{~cm}$ in length folded and $17 \mathrm{~cm}$ in length unfolded.

During the first operation, the prefabricated prosthesis is inserted into the distal aneurysm while the ascending aorta and aortic arch are repaired. During the second operation, a clamp is put on a $2-\mathrm{cm}$ single tube at the proximal side, and then the aneurysm is opened. At the proximal site, the ET is located within the lumen. The 4 stitches are pulled away, and the ET is pulled out. Then the prosthesis is pulled open. The triple prosthesis with 2 folds is stretched as an unfolded, straight, and single graft. Then the residual aneurysm is opened, and a proximal anastomosis is made between the 3-fold ET and the new prosthesis. 


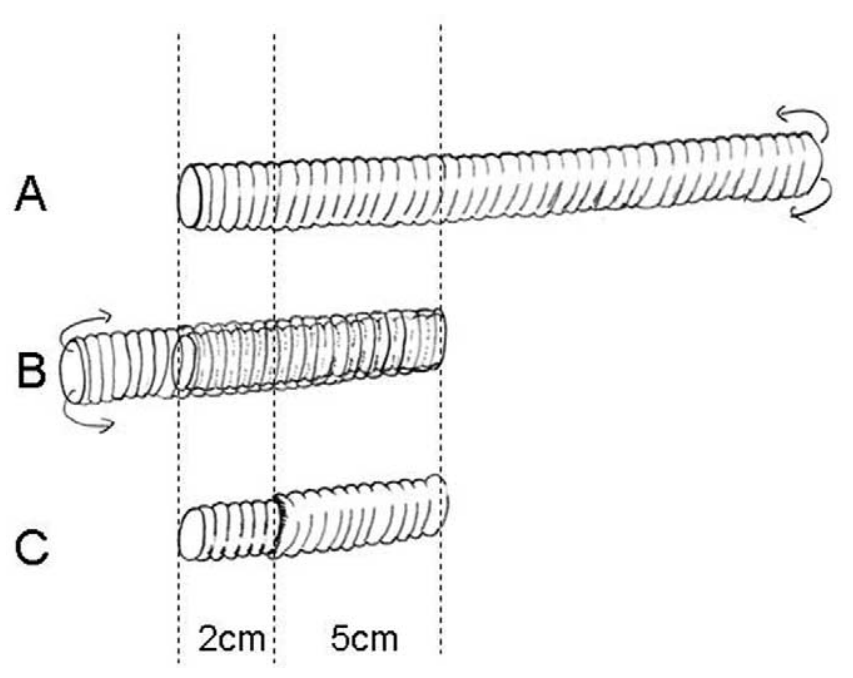

Figure 1. Schema of the 3-fold elephant trunk technique. The 3-fold elephant trunk consists of 2 parts: one 2-cm single tube and another 5-cm triple tube. A, Straight graft; B, folded graft; C, 3-fold graft (2-cm single tube and $5-\mathrm{cm}$ triple tube) with 4 stitches.

\section{Discussion}

he formation of blood clots in the blind pocket between the trunk and the native aneurysmal wall could give rise to thromboembolic events, a long ET could result in thrombotic occlusion of some critical intercostal arteries, or paraplegia could arise. Heinemann and colleagues ${ }^{2}$ have reported that some old clots are often found in blind aortic pockets surrounding the trunk. The longer the trunk, the more stasis in the blind pocket.

The prosthesis was $10 \mathrm{~cm}$ long in the original article. ${ }^{3}$ Schepens and associates ${ }^{4}$ have advocated using prostheses 5 to $10 \mathrm{~cm}$ long.
In other words, to facilitate secondary operations, ETs should be longer. However, to prevent thromboembolic events and paraplegia, ETs should be as short as possible. There is an inconsistency between the 2 requirements. The 3 -fold ET technique can address this inconsistency.

By using this technique, the prosthesis can be made to be less than $10 \mathrm{~cm}$ long. At subsequent operations, the folded prosthesis can be unfolded. Then a triple-length ET graft of up to $17 \mathrm{~cm}$ can be obtained immediately. Use of this type of graft reduces the frequency of thromboembolic events and paraplegia. In addition, the grafts are long enough to facilitate secondary operations.

The original article ${ }^{3}$ indicated that the ET graft was $10 \mathrm{~cm}$ long, and the free end floated freely within the lumen. There was some concern over mechanical complications, such as graft kinking, obstruction, or wrinkled and shrunken ET grafts. These complications could induce a significant pressure gradient within the ET graft. We modeled this problem and, as shown in Figure 2, the inside diameter is sufficient with a 3-fold ET. We also think that freely floating ETs can cause intimal injuries. Ideally, an ET graft should be a little rigid and sufficiently long. The modified technique meets both of these requirements.

The formation of blood clots might be a matter of concern with ET grafts. Carrel and coworkers ${ }^{1}$ have reported that no macroscopic clots were found between the 2 layers of the reversed and bidirectional ET graft. We hypothesize that the blood flow always touches the outside surface of the prosthesis because of the invaginating nature of the prosthesis. Essentially, a prosthesis should permit the passage of blood from the inside. There are many special anticoagulant artifices on the inside surface of the prosthesis. On the contrary, there are no anticoagulant mechanisms on the outside surface of the prosthesis. We concluded that to prevent clot formation, it was most important to force blood to flow continuously along the inside surface of the graft. Therefore the concept of the 3 -fold ET favors an enveloping prosthesis and not an invaginating prosthesis.
A

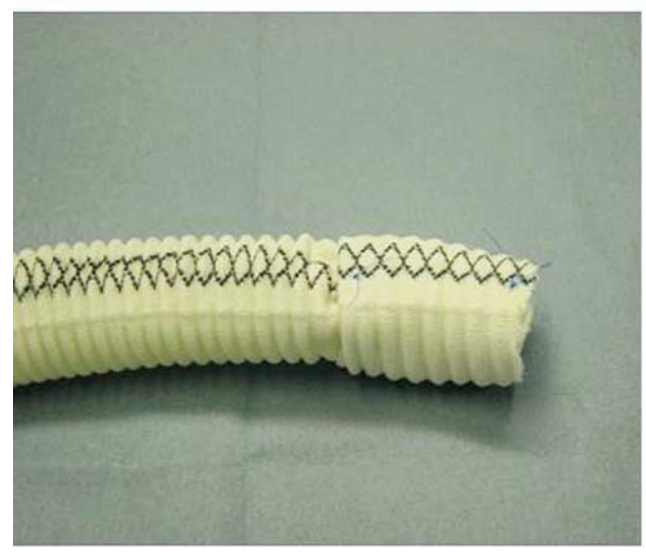

B

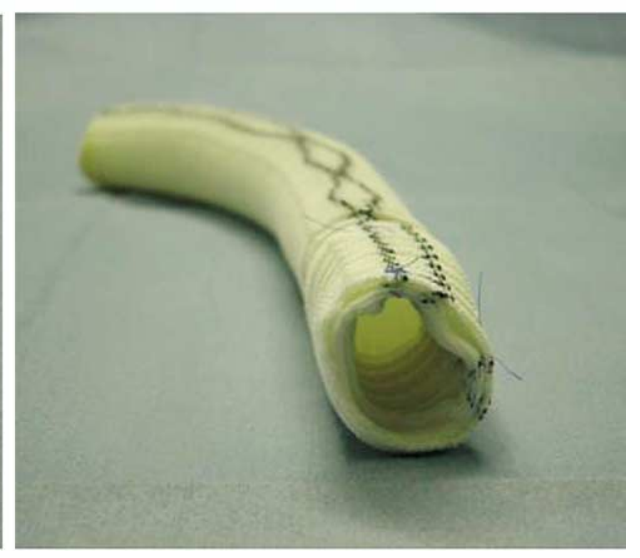

Figure 2. Photographs of the operation. A, An image showing the 3-fold elephant trunk technique. B, Significant stenosis is not seen in the lumen of 3-fold elephant trunk grafts. 


\section{References}

1. Carrel T, Berdat P, Kipfer B, Eckstein F, Schmidli J. The reversed and bidirectional elephant trunk technique in the treatment of complex aortic aneurysms. J Thorac Cardiovasc Surg. 2001;122:587-91.

2. Heinemann MK, Buehner B, Jurmann MJ, Borst HG. Use of the "elephant trunk technique" in aortic surgery. Ann Thorac Surg. 1995;60:2-7.
3. Borst HG, Walterbusch G, Schaps D. Extensive aortic replacement using "elephant trunk" prosthesis. Thorac Cardiovasc Surg. 1983;31: $37-40$

4. Schepens MA, Dossche KM, Morshuis WJ, van den Barselaar PJ, Heijmen RH, Vermeulen FE. The elephant trunk technique: operative results in 100 consecutive patients. Eur J Cardiothorac Surg. 2002;21: $276-81$.

\title{
Acute intrathoracic gastric volvulus after laparoscopic fundoplication: Laparoscopic reduction and repair
}

\author{
Vishwanath Golash, MS, FRCS, Salalah, Sultanate of Oman
}

A

cute gastric volvulus is extremely rare after laparoscopic fundoplication. It is a true surgical emergency, and a delay in diagnosis can lead to fatal complications. Once the diagnosis is confirmed, nasogastric and gastroscopic decompression should be attempted. The surgical treatment involves reduction of the volvulus and prevention of recurrence. We present a patient who had an acute gastric volvulus after a Nissen fundoplication managed laparoscopically.

\section{Introduction}

Gastric volvulus can present acutely or chronically depending on the speed of onset of rotation and the extent of twist. It can also be classified on the basis of the axis of rotation. Organoaxial is the most common and is usually associated with a hiatus hernia. It classically presents with the clinical triad of retching, epigastric pain, and difficulty in passing a nasogastric tube.

\section{Clinical Summary}

A 23-year-old man presented with a 1-day history of increasingly severe upper abdominal and lower chest pain radiating to the back, retching, and abdominal distension. He felt nauseous but was unable to vomit. He had a past history of a laparoscopic Nissen fundoplication a year previously.

On admission, he looked unwell and was pale and tachycardic, with a temperature of $37.5^{\circ} \mathrm{C}$. He had upper abdominal distention and tenderness. His white cell count was 16.5/L, his hemoglobin level was $12 \mathrm{~g}$, and his $\mathrm{C}$-reactive protein concentration was

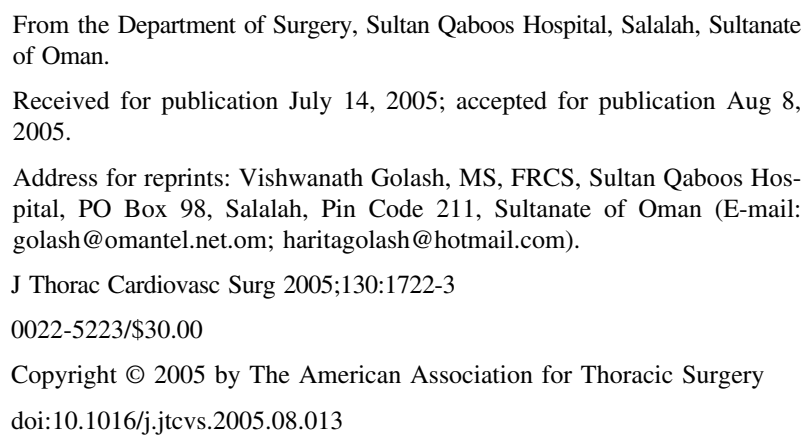

increased at $12 \mathrm{mg} / \mathrm{L}$. The results of the rest of his laboratory investigations, including the serum amylase and troponin measurements and electrocardiography, were normal.

The chest radiographs demonstrated 2 air-fluid levels within

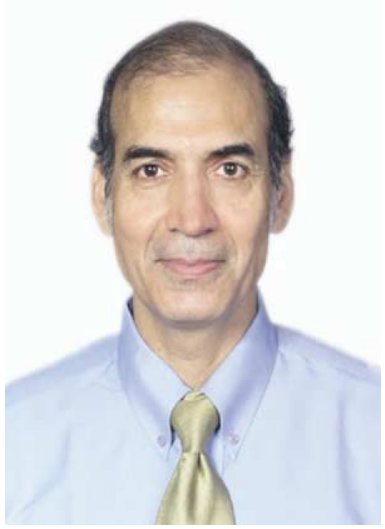

Dr Golash the chest at differing heights and a raised left hemidiaphragm. These findings were suggestive of a gastric volvulus. An urgent contrast-enhanced computed tomographic scan of his chest and abdomen showed 2 air-fluid levels in the posterior mediastinum (Figure 1). ${ }^{1}$ In lower cuts of the computed tomographic scan, a transition line was seen between the 2 air-fluid levels representing the site of the volvulus. In addition, in the lower cuts the left crus was not seen, suggesting the possibility of a rupture of the left crus.

An emergency gastroscopy revealed a large blood clot within the stomach, although no definite site of bleeding could be identified. The mucosa was inflamed at the previous site of fundoplication. The pyloric end of the stomach could not be reached. The stomach was decompressed at the end of the procedure, and it was believed that the bleeding was possibly caused by mucosal ischemia. A barium study confirmed the volvulus of the intrathoracic herniated part of the stomach. Barium reached the pyloric end of the stomach after 2 hours. He was taken for emergency surgical intervention that afternoon.

The diagnosis was confirmed at laparoscopy. A mark at the site of constriction was visible as a ring on the greater curvature of the stomach, suggesting that partial reduction of the volvulus had occurred and possibly after gastroscopy. Along with the stomach, the caudate lobe of the liver had also migrated into the chest. The herniated part of the stomach and liver was reduced into the abdomen. ${ }^{2}$ After adequate adhesiolysis in and around the hiatus and the posterior mediastinum, the esophagus was skeletonized, and an adequate intra-abdominal length of esophagus was freed. The hernial sac in the posterior mediastinum housing the volvulus was completely excised. The previous cruroplasty had torn apart with severe anatomic disruption. The hiatus was about $8 \mathrm{~cm}$ in diameter, and it was not possible to 